\title{
Imaging of GaAs nanowire using combined aberration-corrected TEM/STEM and exit wave restoration
}
L. Y. Chang, $* * * * * *$
S. Lazar,**
B. Bártová, $* * *$
G. Botton,*
C. Hébert*** and A. Fontcuberta i Morral****

* Canadian Centre for Electron Microscopy (CCEM), McMaster University, 1280 Main Street West, Hamilton, ON, Canada L8S 4M1

** FEI Electron Optics, 5600 KA Eindhoven, The Netherlands

*** LSME, École Polytechnique Fédérale de Lausanne, CH-1015 Lausanne, Switzerland

**** LMSC, École Polytechnique Fédérale de Lausanne, CH-1015 Lausanne, Switzerland

****** Monash Centre for Electron Microscopy, Monash University, Vic 3800, Australia

Semiconductor nanowires are of high interest as potential building blocks for future optical and electronic devices, such as high mobility transistors, bright LEDs and solar cells [1]. Nanowire growth has been achieved by the use of alternative metals such as aluminum, titanium and copperthat dope or have much lower solubility-or by simply avoiding the use of a catalyst. The synthesis was carried out in a GenII molecular beam epitaxy (MBE) machine. The growth of the wires is achieved from gallium/indium alloy droplets that are nucleated in situ [2]. For the TEM measurements, the wires were mechanically removed from the substrate and transferred with acetone droplet to a holey-carbon grid.

High-angle annular dark field (HAADF) STEM mode is Z-contrast dominant, and its contrast is generally regarded to be directly interpretable, under suitable probe and specimen conditions [3]. On the other hand, high-resolution electron microscopy (HREM) images suffer from aberrations introduced by the objective lens, and these are generally not directly interpretable. Phase of the specimen exit wave function from a focal series of HREM images [4] gives an aberration free, complex exit wave function. Its contrast can be interpreted to be approximately proportional to the projected potential, given the object is reasonably thin. The signal to noise ratio in the restored phase is greatly improved compared to a single electron micrograph, making direct imaging of atoms on and near surfaces of nanowires possible.

Figure 1 shows an overview image of investigated sample. The nanowire diameter varies from 60 to $90 \mathrm{~nm}$. Each wire contains different amount of defects. The wires present a zinc blende structure as well as wurtzite structure. Twinned structures and stacking faults can be clearly seen along the nanowire in the aberration-corrected HAADF STEM image, as shown in Figure 2. The aberrationcorrected high-resolution TEM image shown in Figure 3, on the other hand, gives detailed features of the surface. Figure 4 shows the restored phase of a surface region of the nanowire shown in Figure 3. Note that the dumbbells of $\mathrm{Ga}$ and As are resolved, and the contrast between Ga and As can be clearly distinguished. A mirror twin can be directly visualized in the phase.

References

[1] W. Lu et al. Proc. Natl Acad. Sci. 102 (2005) 10046-51.

[2] C. Colombo et al, Phys. Rev. B 77 (2008) 155326

[3] S. J. Pennycook et al. Nature 336 (1988) 565.

[4] R. R. Meyer et al. Ultramicroscopy 92 (2002) 89. 

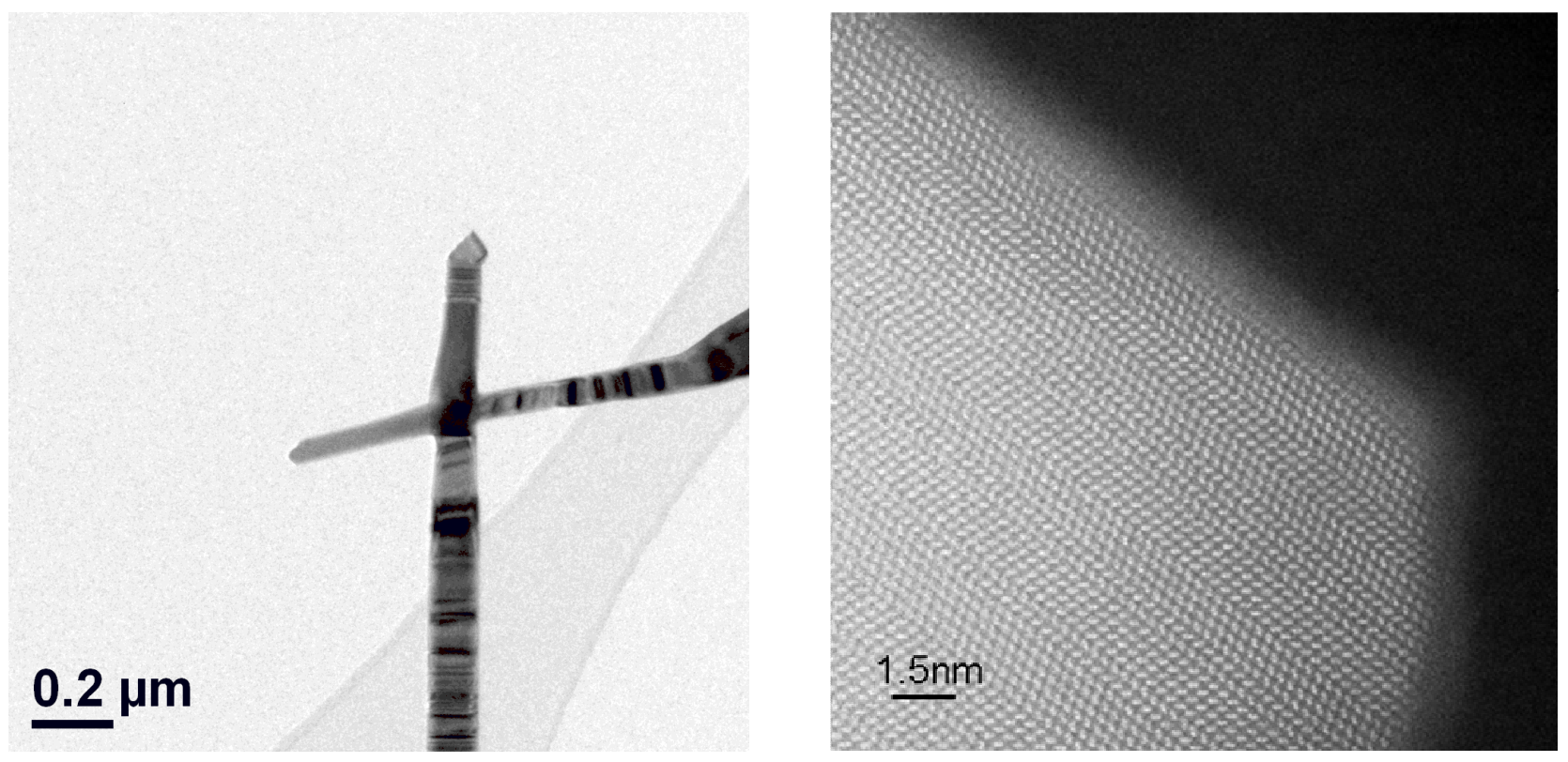

FIG. 1. Overview image of examined sample. FIG. 2. Aberration-corrected HAADF STEM image of a GaAs nanowire.

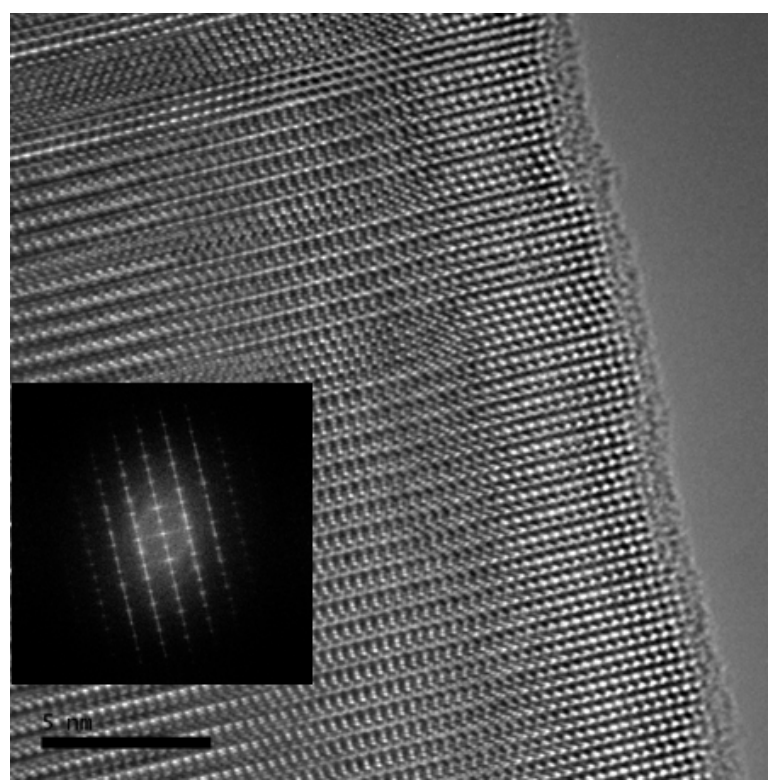

FIG. 3. A member of the aberration-corrected HRTEM focal seires of a GaAs nanowire.

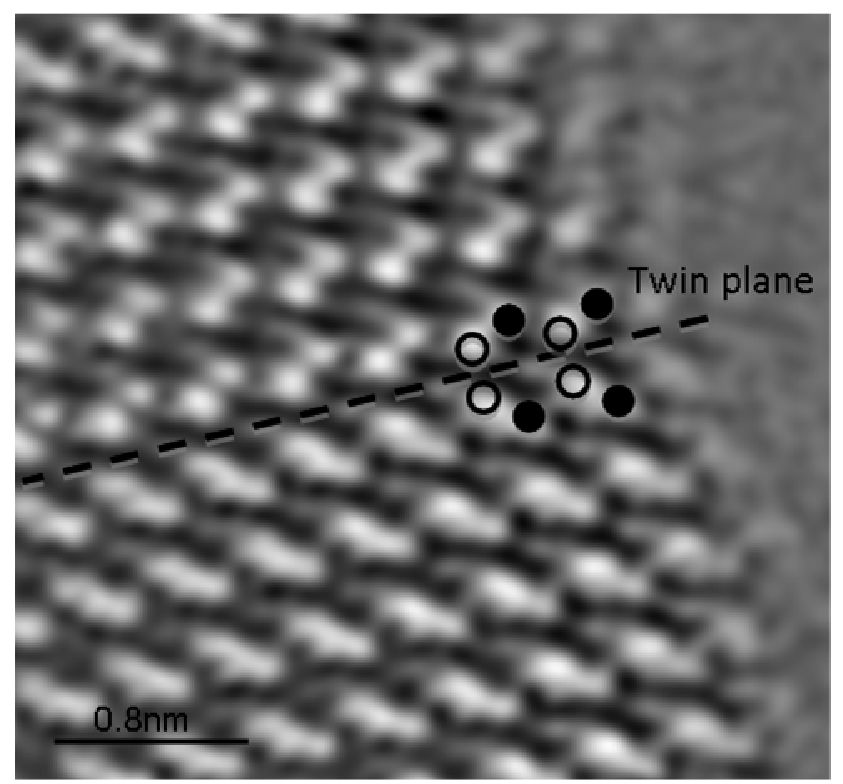

FIG. 4. Restored phase of the exit wave function of a GaAs nanowire in a small region near the edge of the nanowire. 